\title{
Passive control of attachment in legged space robots
}

\author{
Alessandro Gasparetto $^{\mathrm{a}}$, Renato Vidoni ${ }^{\mathrm{a}}$ and Tobias Seidl ${ }^{\mathrm{b} *}$ \\ ${ }^{a}$ DIEGM, Department of Electrical, Management and Mechanical Engineering, University of Udine, Via delle Scienze, Udine, Italy; \\ ${ }^{b}$ Advanced Concepts Team, European Space Agency, AZ Noordwijk, The Nederlands
}

(Received 12 August 2008; final version received 20 March 2009)

\begin{abstract}
In the space environment the absence of gravity calls for constant safe attachment of any loose object, but the low-pressure conditions prohibit the use of glue-type adhesives. The attachment system of freely hunting spiders, e.g. Evarcha arcuata, employs van der Waals forces and mechanical interlocking. Furthermore, detachment is achieved passively and requires little force. Hence, the spider serves as a model for a versatile legged robot for space applications, e.g. on the outer surface of a space station. In this paper, we analyse the dry attachment systems of E. arcuata and geckos as well as the kinematics of freely hunting spiders. We generalise the results of biological studies on spider locomotion and mobility, including the major movement and the position constraints set by the dry adhesion system. From these results, we define a simplified spider model and study the overall kinematics of the legs both in flight and in contact with the surface. The kinematic model, the data on spider gait characteristics and the adhesion constraints are implemented in a kinematic simulator. The simulator results confirm the principal functionality of our concept.
\end{abstract}

Keywords: spider; adhesion; multi-body; kinematics; locomotion

\section{Introduction}

The space environment is technically highly challenging and any space-adapted autonomous system has to be conceived accordingly carefully (Bellingham and Rajan 2007). Since almost all spacecraft are situated in rather remote locations during duty and hence cannot be accessed easily for repair, absolute reliability is mandatory. In consequence, technology should be as simple as possible to fulfill the desired task. In addition to that, energy supply is extremely limited, putting high selection pressure on technology with high energy consumption. In the view of these constraints, we investigate potential alternatives for attachment - in the absence of gravity, reliable attachment is essential for any kind of activity. In the present account, we focused on means of attachment for robots working outside a space station.

In current applications, specifically mounted handles or rails facilitate grabbing but also impose kinematic constraints restricting the dexterity in operations. Ideally reliable attachment to non-specific surfaces would allow for an extended action range and versatility of 'legged' space robots. Commonly available solutions for climbing smooth surfaces are, e.g. suction cups or magnetic feet (Yano et al. 1997; Hirose and Kawabe 1998; La Rosa et al. 2002; Xu and Ma 2002). However, they require either particular predefined surfaces and/or exhibit high-energy consumption when in use and hence should be avoided in the space environment. On the other side, high motoric versatility including reliable attachment and easy detachment on a broad range of surfaces is a feature of some animals including freely hunting spiders, Evarcha arcuata. These animal models - insects, spiders, tree frogs and geckos - are the focus of research on biological attachment mechanisms (Menon et al. 2004; Menon and Sitti 2005; Unver et al. 2006; Kim et al. 2007) and have seen a huge advance not only in terms of analysis but also transfer to first working prototypes.

Among the broad variety of biological attachment mechanisms (Nachtigall 1974), the ones employing socalled reversible 'dry attachment' appear as ideal models for space applications. Dry adhesion is reported in both geckos and spiders (Autumn et al. 2000; Kesel et al. 2003, 2004; Gao et al. 2005; Niederegger and Gorb 2006). In both cases, the feet adhere to smooth surfaces via a multitude of tiny hairs (setulae). They exploit an intermolecular attraction - van der Waals forces - without requiring sticky fluids as mediators. In consequence, these attachment mechanisms principally qualify for application in non-atmospheric, low-pressure environments. The spider attachment system works - in contrast to the muscularly mediated one of the geckos - completely passively; it is only controlled by the movement of the animal's leg and therefore control of attachment can be integrated into the leg's movement control. Starting from these basic assumptions,

*Corresponding author. Email: tobias.seidl@esa.int 
we analysed the spiders' strategies to attach to smooth surfaces to conceptualise a technical solution principally able to walk freely on the outer surface of a space station. In the present account, we report on our analysis of spiderleg kinematics, derive a simplified and generalised model, which we then evaluate in a simulation environment.

The results of the present work show that a legged robot using biomimetic, asymmetric adhesives can control attachment to various types of surfaces merely via the kinematics of the legs. In principle, the robotic concept is designed to cope with non-coplanar surfaces or multiple objects. The present system does not require dedicated adhesion control.

\section{Passive control of attachment in spiders}

\subsection{Morphology and ecology}

Spiders (Araneae) belong to the taxonomic phylum of arthropods together with, e.g. insects and scorpions, and hence have an exoskeleton in common with them. The spider body consists of the cephalothorax from which four leg pairs and the organ-bearing abdomen emerge. Each leg is composed of seven segments, starting with the coxa connected to the thorax followed by trochanter, femur, patella, tibia, metatarsus and finally the ground-touching tarsus (Foelix 1996) (Figure 1). The types of inter-segmental limb joints are highly conservative among the different spider species and mostly show variations in angular working ranges. Most of the joints are bicondylar and feature large angular movements around one axis of rotation. Mono-

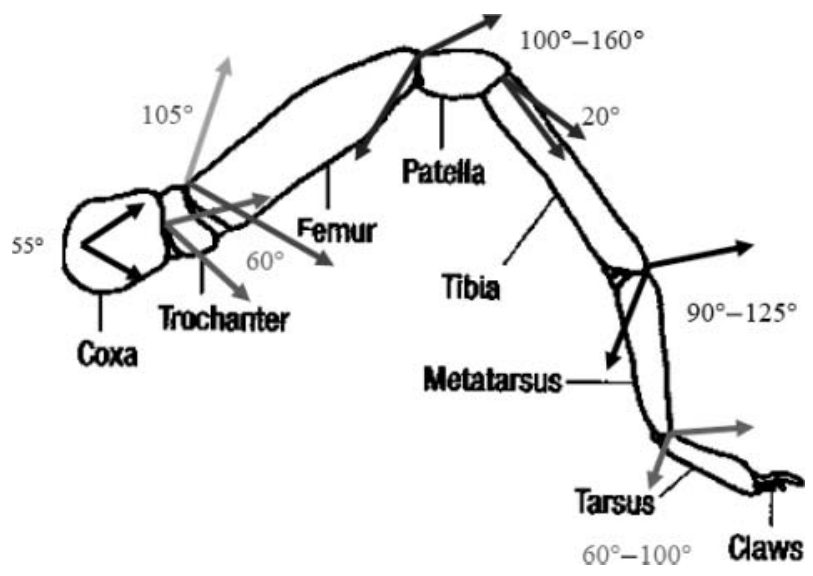

Figure 1. Ranges of motion for joints in the vertical $Y-Z$ plane. condylar joints have multiple axes of movement and usually perform little movements during locomotion (Shultz 1987; Sensenig and Shultz 2002, 2004). A peculiarity of the spider leg compared to most other known articulated legs is the $\mathrm{C}$-shaped configuration. While most known articulated legs are arranged in a $\mathrm{Z}$ shape and are actuated by antagonistic muscle-tendon systems, the extension of both the femur-patella joint and tibia-metatarsus joint is achieved via a hydraulic system.

The ecology, i.e. the habitat and feeding habits of each single species, has effect on parts of the morphology, especially limb geometry and tarsi. On the tarsi of entirely webliving spiders we find prominent claws, which are used to handle threads, and only small scopulae to attach to smooth surfaces. However, exclusively freely hunting spiders have small claws but prominent scopulae, which they use to cling to inclined surfaces and to cope with dynamic loads appearing during prey-catching and jumping. Depending on the species and their natural behaviour the adhesion system can be found on the tip of the tarsus (e.g., E. arcuata (Kesel et al. 2003, 2004)) or on the overall ventral side of the tarsus and/or metatarsus limbs (e.g. Aphonopelma seemanni and Cupiennius salei (Niederegger and Gorb 2006)). The scopulae of spiders are composed hierarchically by setae and setulae forming more than $10^{6}$ terminal ends per leg and are brought into contact with the substrate (Kesel et al. 2003, 2004). Adhesion between one of these terminal ends and the substrate is achieved via intermolecular van der Waals forces without the mediating presence of any sticky fluids (Kesel et al. 2003, 2004). Attachment of the entire scopula to a surface is controlled via the dynamics during contact establishment between scopula and substrate. In consequence, a statistical distribution of single setula-substrate contacts establishes reliable grip. Detachment on the other side is achieved on an almost zero-cost basis by exploiting the asymmetry of the setulae terminal ends via dedicated kinematics at the end of the stepping cycle.

\subsection{Geometry and function of spider limbs}

In the following, we describe the major characteristics of geometry and function of spider limbs as reported by Ellis (1943), Parry (1957), Shultz (1987), Foelix (1996), and Karner (1999) as far as they were used for the development of our simplified model.

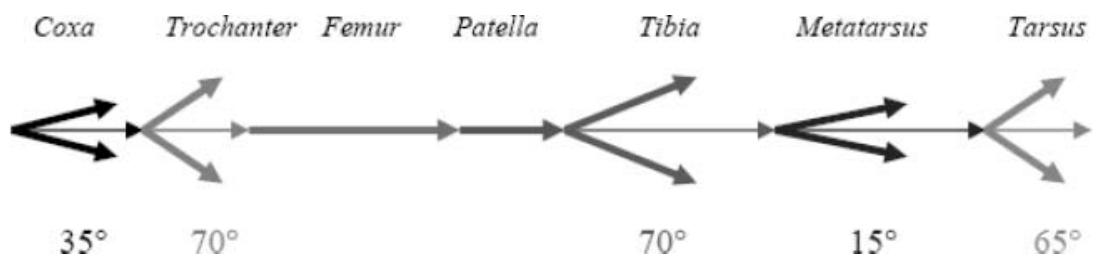

Figure 2. Ranges of motion for joints in the horizontal $X-Y$ plane. 
The connection between the leg and the body, the thorax-coxa joint, can move both in the horizontal and vertical plane (Foelix 1996). It can be characterised either as a ball-and-socket (or spheroid) joint with 3 degrees of freedom (DOF) or as a 2 DOF saddle joint with two axes of rotation orthogonal, respectively, to the vertical and horizontal plane. The following joint connects coxa and trochanter. It is as a ball-and-socket joint actuated by a muscle-tendon system (Parry 1957). According to Shultz (1987), it has significant rotation movements during locomotion, while according to Foelix (1996), it is a saddle joint with 2 DOF. The trochanter-femur joint is a bicondylar joint actuated by four muscles and hence displays a 1 DOF revolute pair with movements only in the vertical plane. The femur-patella joint connects the long femur and the relatively small and short patella. While flexion is achieved by muscle-tendon units, extension is enabled by a hydraulic mechanism (Ellis 1943; Foelix 1996). Being a bicondilar joint, we model it as a hinge joint with 1 DOF. The patella-tibia joint has a high range of movement in the horizontal plane and only limited in the vertical plane (Parry 1957; Foelix 1996) and in consequence it can be modelled either as a hinge joint or a universal joint (2 DOF) with very limited movement in the vertical plane. The tibia-metatarsus joint-with the metatarsus being one of the longest limb segments-is actuated by muscle-tendon systems during flexion and by hydraulics during extension. Different angles of movements are reported depending on the species analysed. The movements are relatively free in the vertical and very limited in the horizontal plane. Hence, it is possible to assume this joint either as a hinge joint or as a universal joint with very limited motion on the horizontal plane, in contrast with the patella-tibia joint. The most distal joint is the metatarsustarsus joint; it connects the metatarsus and the short tarsus limb where the claws and the scopula are attached. This joint cannot be directly actuated as there are no muscles directly associated with it; the layout of the long tendons on the metatarsus is responsible for the active movement. According to Parry (1957), an articular condyle is absent and hence this joint can be regarded as a universal joint with 2 DOF.

As mentioned above, spider limbs vary with species reflecting each the animal's lifestyle. Hence, it is difficult to derive a rule for the size of limbs or limb segments apart from general relationships. Two close and rather conservative ratios are found following data of Yoshida et al. (2000) and Huang (2004) and serve for generalisation: the ratio femur/patella+tibia is between 0.85 and 1.05 ; the femur/metatarsus+tarsus ratio is between 0.75 and 0.8 . Following the above-made considerations, we composed firstly a model of a spider leg with the ranges of motion for each joint in the vertical $(Y-Z)$ and in the horizontal $(X-Y)$ planes (compare Figures 1 and 2), and, secondly, a general kinematic scheme of the leg (Figure 3).

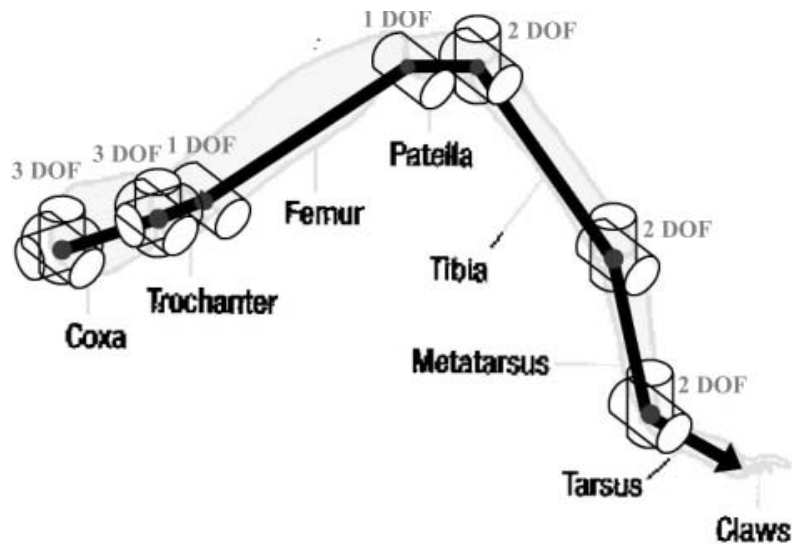

Figure 3. General kinematic scheme for a spider leg.

\subsection{Adhesion system}

In spiders, the tarsi bear two distinct attachment systems used to adhere to the substrate. The tarsal claws are prominent hooks and serve to interlock on rough surfaces and thread handling (in web-living spiders). The second system, the scopula, attaches to smooth surfaces, which do not permit mechanical interlocking. The scopula is composed of a set of hairs, the setae; on the ventral side of which tiny hairs, the setulae, emerge. When brought into contact with the substrate, the flattened and triangular-shaped terminal ends of the setulae establish adhesion via intramolecular forces, the van der Waals forces. The ramified hierarchical arrangement of setae and setulae allows to bring a sufficiently high number of terminal ends into contact to generate reliable attachment for the animal. Attachment is achieved via a statistical process as not all possible contacts are established during touchdown. In the jumping spider $E$. arcuata, the maximum possible adhesive force per setula was determined to be $41 n N$. When considering the estimated 624,000 setulae in the whole animal, the total adhesive force reaches $2.56 \times 10^{-2} \mathrm{~N}$ or a maximum safety factor, i.e. the ratio between the total adhesive force and the spider weight, of 173 (Kesel et al. 2004). This theoretical value, however, reflects on the dynamic lifestyle of the animal: it does not only walk on vertical surfaces, but also jumps and catches prey and hence needs to establish higher attachment forces than necessary for plain walking (Betz and Kölsch 2004).

Contrary to the gecko's attachment system, the spider's scopula is not actuated via, e.g. muscles and joints. During attachment, compliance is achieved through elastic and damping properties of both material and hierarchical structure. Detachment of the single seta, however, is achieved via the angle between tarsus and substrate. According to the work on gecko-setulae by Gao et al. (2005) using finite element modelling, there are two principal ways of breaking the contact: the seta (i) slides off when the angle between 
seta and substrate reaches $0^{\circ}$ and (ii) detaches at an angle approaching $90^{\circ}$. The adhesive force is maximal when the angle between seta and substrate is near $30^{\circ}$. Moreover, it has been demonstrated that in a real gecko there is an enhance of adhesion if a small proximal movement (5-10 $\mu \mathrm{m})$ is added to a normal preload in the attaching phase (Autumn et al. 2000). Extrapolating from the geckodata to the spider attachment system, the proximal and distal movements of the leg are crucial to technically reproduce the passive control in a legged walker. The angle of adhesion of the setal structure, which is on the tip of the tarsus, can be directly related to the approaching angle of the last limb of the leg's chain. Therefore, the approaching angle between the last limb of the leg and the surface has to be suitably controlled to lead to a functional implementation of the adhesion and detachment phases.

\section{Mobility analysis and model building}

During a stepping cycle each leg passes through two mechanically different situations, which need to be considered for a kinematic and mobility analysis: first, the free-flight configuration where the leg is not in contact with the substrate, and second the contact configuration with the leg touching the substrate. During free-flight configuration, the leg is lift up from the ground and swung forward to initiate a new step. Since the leg is only coupled to the central body/thorax we see an open-chain; the position of the body is defined by those other legs, which are in contact with the substrate. The position of the body can be considered as known and serves as the base frame of the kinematic chain; the tip of the tarsus serves as the end-effector. When the tarsus is in contact with the substrate, during stance phase, we are confronted with a closed-chain. The point of contact of each ground-touching leg becomes a fixed point and is linked to the frame - in this case the substrate. For a kinematic analysis, we have to consider the free-flight configuration as an open-chain manipulator with seven joints emanating from the body and ending at the tarsus, while during ground contact, the point of contact is to be modelled with an additional spherical wrist. The mobility of each leg is described by the Kutzback equation:

$$
d=6 \cdot(n-1)-\sum_{i=1}^{j}\left(6-f_{i}\right)
$$

where $n$ stands for the number of links, $j$ the number of joints, $f_{i}$ the DOF of each joint and $d=$ DOF per leg. During free flight $d$ reaches a value of $14 \mathrm{DOF}$, while it extends to $d=17$ during ground contact (compare Table 1).

The overall mobility $M$ of the entire structure can be calculated with the same Kutzback equations by taking into account the contribution of all legs and their respective condition by considering $n=$ (no. of legs in contact) $\times$ (no. of joints of each leg) + body + substrate. It depends upon the number of legs in contact $(L)$ and the number of DOF per leg in contact $(d)$. With fewer than 6 DOF per leg $(d<6)$ the overall mobility $M$ decreases with an increase of legs in ground contact. On the other hand, for legs with more than 6 DOF $(d>6)$ any increase of rested legs leads to an augmentation of the system's overall mobility. With exactly 6 DOF $(d=6)$, the mobility of the system is 6 regardless of the number of legs in contact with the substrate. In this case, the spider's body can be moved in all directions and with all orientations. For every chosen position and orientation of the body, a finite number of configurations for every link is allowed. If the mobility $M$ equals zero at least one leg must be lifted off the substrate to move the body; when the overall mobility exceeds the value of six $(M>6)$ at least one leg pair can assume infinite values at a chosen position and orientation of the body. Hence, taking into account that the spider can control the position and orientation of its body and in the meantime the adhesion (via the angle between the tarsus and the substrate) of each leg, the number of DOF per leg has to be greater than 6 .

Table 1. Mobility of the spider system in dependence of degrees of freedom of a single leg and the number of legs in contact with the substrate.

\begin{tabular}{|c|c|c|c|c|c|c|c|c|c|c|c|c|c|c|c|}
\hline \multirow{2}{*}{$\begin{array}{l}\text { Legs in } \\
\text { contact }\end{array}$} & \multicolumn{15}{|c|}{ Degrees of freedom of single leg } \\
\hline & 3 & 4 & 5 & 6 & 7 & 8 & 9 & 10 & 11 & 12 & 13 & 14 & 15 & 16 & 17 \\
\hline 1 & 3 & 4 & 5 & 6 & 7 & 8 & 9 & 10 & 11 & 12 & 13 & 14 & 15 & 16 & 17 \\
\hline 2 & 0 & 2 & 4 & 6 & 8 & 10 & 12 & 14 & 16 & 18 & 20 & 22 & 24 & 26 & 28 \\
\hline 3 & & 0 & 3 & 6 & 9 & 12 & 15 & 18 & 21 & 24 & 27 & 30 & 33 & 36 & 39 \\
\hline 4 & & & 2 & 6 & 10 & 14 & 18 & 22 & 26 & 30 & 34 & 38 & 42 & 46 & 50 \\
\hline 5 & & & 1 & 6 & 11 & 16 & 21 & 26 & 31 & 36 & 41 & 46 & 51 & 56 & 61 \\
\hline 6 & & & 0 & 6 & 12 & 18 & 24 & 30 & 36 & 42 & 48 & 54 & 60 & 66 & 72 \\
\hline 7 & & & & 6 & 13 & 20 & 27 & 34 & 41 & 48 & 55 & 62 & 69 & 76 & 83 \\
\hline 8 & & & & 6 & 14 & 22 & 30 & 38 & 46 & 54 & 62 & 70 & 78 & 86 & 94 \\
\hline
\end{tabular}

The chosen simplified leg with 7 DOF and the corresponding mobility of the spider robot are marked in italic typesetting. 

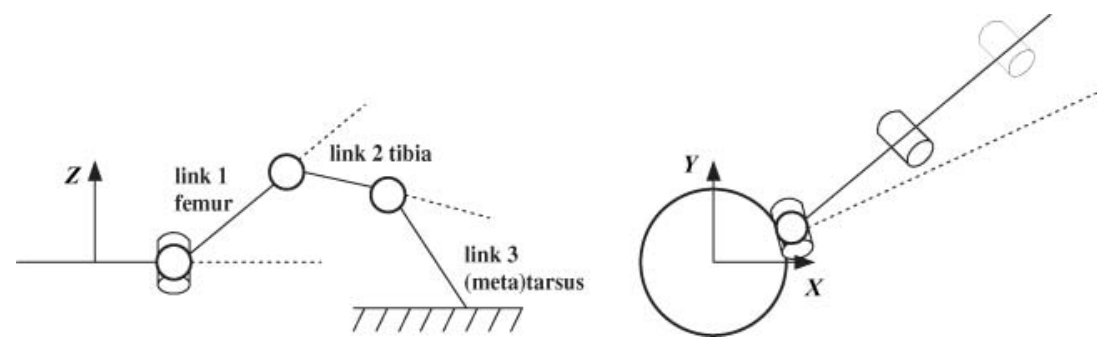

Figure 4. Simplified spider's leg model.

The overall mobility of the spider model reaches 94 DOF ( $d=17, L=8)$ in resting condition (i.e., all legs in contact with these substrate), and $50 \mathrm{DOF}(d=17, L=$ 4 ) with four legs in the air. Taking into account that only $6 \mathrm{DOF}$ are required to define position and orientation of a rigid body in space, the exceeding mobility serves for tasks different from walking, such as grasping and manipulating of objects, as well as hunting and active tactile sensing. In the present account, however, we focus on replicating walking, including control of attachment to the substrate. In consequence our targeted locomotion system will have drastically reduced kinematic complexity compared to the spider model. However, in order to comply with the peculiarities of the attachment system - the control of the tarsus-substrate angle - our target model has 7 DOF per leg.

Finally, the chosen model has eight uniform legs radially collocated around a circular central body. It does not reflect ecologically influenced variation in leg length or existence of an abdomen as found in the biological models, and hence it is rotationaly symmetric. The uniform leg contains three links and three joints, one universal (2 DOFs) and two revolute (1 DOF for each) pairs (Figure 4). In the open-chain configuration the leg has $4 \mathrm{DOF}$; in the contact configuration seven, considering the tarsus-substrate point as a virtual spherical joint with 3 DOF.

\section{Kinematics}

In the following, we analyse and describe the kinematics required to fulfill the tasks of locomotion and reversible adhesion. Both position and orientation of the body shall be evaluated as well as approaching angles between tarsus and substrate by solving the kinematic problem. As mentioned above, both conditions - with and without ground contact of the tarsus - have to be treated separately. For the free-flight kinematics, the target is to solve the direct and inverse kinematic problem for the open-chain configuration to control the (meta)tarsus of each leg by considering the body position known and fixed. The targets to solve in the contact kinematics are the direct and inverse kinematics of the closed-chain to control the position and orientation of the body. The positions of the contact points between the supporting legs (legs in contact with the substrate) and the substrate are considered as fixed. The overall kinematics of the system can then be solved by combining the free flight and the contact kinematics.

\subsection{Free-flight kinematics}

By using the Denavit-Hartenberg (DH) convention (Sciavicco and Siciliano 2001), the coordinate reference systems of the model are fixed as shown in Figure 5 and Table 2 starting from the body reference system.

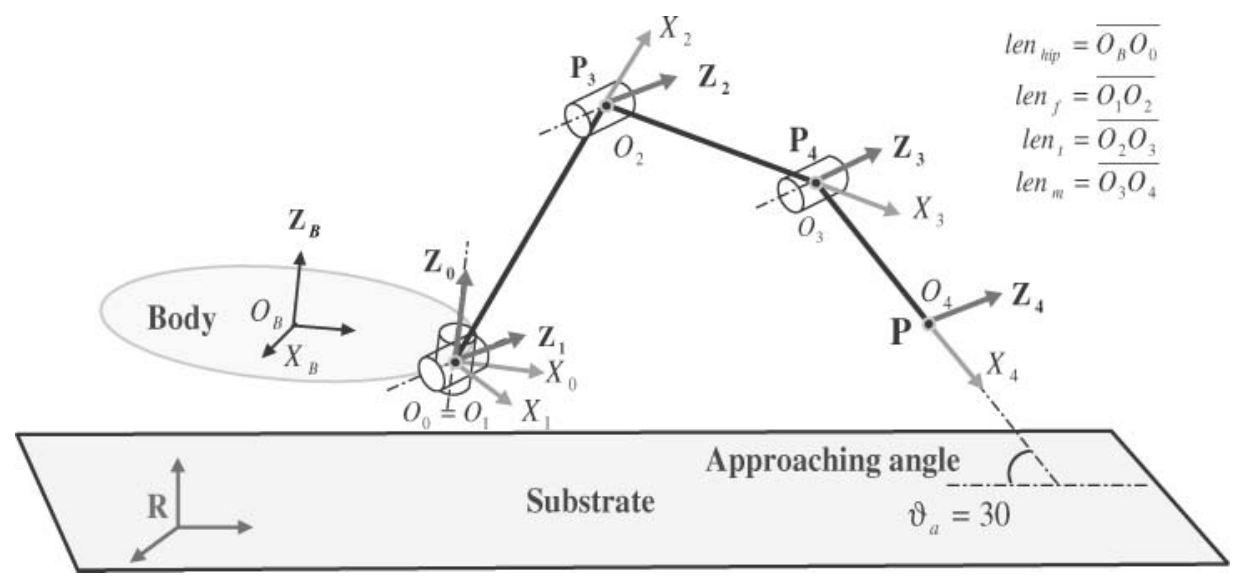

Figure 5. Spider's leg model with the DH coordinate systems. 
Table 2. Denavit-Hartenberg parameters of the spider leg.

\begin{tabular}{lcccc}
\hline & $d_{i}$ & $\theta_{i}$ & $a_{i}$ & $\alpha_{i}$ \\
\hline Body $\rightarrow 0$ & 0 & $\theta_{0}$ & len $_{\text {hip }}$ & 0 \\
$0 \rightarrow 1$ & 0 & $\theta_{1}$ & 0 & $\frac{\pi}{2}$ \\
$1 \rightarrow 2$ & 0 & $\theta_{2}$ & len $_{f}$ & 0 \\
$2 \rightarrow 3$ & 0 & $\theta_{3}$ & len $_{t}$ & 0 \\
$3 \rightarrow 4$ & 0 & $\theta_{4}$ & len $_{m}$ & 0 \\
\hline
\end{tabular}

These parameters allow to express and find a transformation from the end to the base of a manipulator with a systematic technique following (Sciavicco and Siciliano 2001).

\subsubsection{Direct kinematics}

The position and orientation of the tip of the (meta)tarsus can be easily computed once the values of each pair are known. Defining $\mathbf{A}_{i}^{j}$ as the rototranslation matrix between the joint $j$ and $i$, the direct kinematics equation becomes (Figure 5):

$$
\mathbf{A}_{4}^{R}=\mathbf{A}_{B}^{R} \cdot \mathbf{A}_{0}^{B} \cdot \mathbf{A}_{1}^{0} \cdot \mathbf{A}_{2}^{1} \cdot \mathbf{A}_{3}^{2} \cdot \mathbf{A}_{4}^{3},
$$

where the matrix $\mathbf{A}_{B}^{R}$ is known for hypothesis and the $\mathbf{A}_{0}^{B}$ is defined once the leg is chosen. As the relation between the $B$ and 0 coordinate systems is fixed, the rototranslation matrix between the coordinate system 4 and 0 is

$$
\mathbf{T}_{4}^{0}=\mathbf{A}_{1}^{0} \cdot \mathbf{A}_{2}^{1} \cdot \mathbf{A}_{3}^{2} \cdot \mathbf{A}_{4}^{3}=\left[\begin{array}{lll|l}
\mathbf{a}^{0} \mathbf{n}^{0} \mathbf{s}^{0} & \mathbf{p}^{0} \\
\hline 0 & 0 & 0 & 1
\end{array}\right],
$$

and the position of the end-effector of the chain $\mathbf{p}^{0}$ and the approaching vector $\mathbf{a}^{0}$ are defined.

\subsubsection{Inverse kinematics}

The inverse kinematics problem solution, i.e. considering the body as fixed and finding the values of the joint pairs to bring the tip of the (meta)tarsus to a defined position and orientation, can be solved by looking at the particular configuration of the leg system (Figure 5). The first $\left(\theta_{1}\right)$ revolute pair is the only one that works in the $X Y$ plane of the body reference system. By defining $\mathbf{P}^{0}$ as

$$
\mathbf{P}^{0}=\left[p_{x}^{0}, p_{y}^{0}, p_{z}^{0}, 1\right]^{\mathrm{T}},
$$

for $\theta_{1}$ holds

$$
\theta_{1}=a \tan 2\left(p_{y}^{0}, p_{x}^{0}\right)
$$

The residual planar manipulator with three links (Figure 4) is made of the 2, 3 and 4 joints that realise an R-R-R dyad. Hence, the inverse kinematics can be analytically solved. Defining $\phi=\theta_{2}+\theta_{3}+\theta_{4}$ the overall rotation on the $\mathbf{Z}$ axis of the joint 2 coordinate system, the position of the center of the 4th revolute pair $\mathbf{P 4}$ becomes

$$
\mathbf{P 4}=\left[p 4_{x}, p 4_{y}, p 4_{z}, 1\right]^{\mathrm{T}}=\mathbf{p}^{0}-\operatorname{len}_{m} \cdot\left[\mathbf{a}^{0}, 1\right]^{\mathrm{T}},
$$

where $\mathbf{p}^{0}$ and $\mathbf{a}^{0}$ are known once defined the target position and the approaching angle. For $\theta_{3}$ holds

$c_{3}=\frac{p 4_{x}^{2}+p 4_{y}^{2}+p 4_{z}^{2}-\operatorname{len}_{f}^{2}-\operatorname{len}_{t}^{2}}{2 \cdot \operatorname{len}_{f} \cdot \operatorname{len}_{t}}, \quad s_{3}= \pm \sqrt{1-c_{3}^{2}}$.

Once $\theta_{3}$ has been computed, $\theta_{2}$ can be found after some algebra:

$$
\begin{gathered}
c_{2}=\frac{\left(\operatorname{len}_{f}+\operatorname{len}_{t} \cdot c_{3}\right) \cdot \sqrt{p 4_{x}^{2}+p 4_{y}^{2}}+\operatorname{len}_{t} \cdot s_{3} \cdot p 4_{z}}{p 4_{x}^{2}+p 4_{y}^{2}+p 4_{z}^{2}}, \\
s_{2}=\frac{\left(\operatorname{len}_{f}+l e n_{t} \cdot c_{3}\right) \cdot p 4_{z}-l e n_{t} \cdot s_{3} \cdot \sqrt{p 4_{x}^{2}+p 4_{y}^{2}}}{p 4_{x}^{2}+p 4_{y}^{2}+p 4_{z}^{2}},
\end{gathered}
$$

$\theta_{4}$ solutions can be found by considering that

$$
\theta_{4}=\phi-\theta_{2}-\theta_{3}
$$

All the unknowns are found and the inverse kinematic problem is solved.

\subsection{Contact kinematics}

When a leg touches the substrate, the contact can be modelled as a virtual spherical joint that closes the kinematic chain between the (meta)tarsus and the surface. Hence, additional coordinate reference systems and DH parameters have been fixed to characterise the overall problem (Figure 6 and Table 3).

From the mobility analysis, the leg structure has 7 DOF and the overall spider model admits $6+n$ DOF, where $n$ is the number of legs in contact with the surface. Hence, for every leg, $\infty^{1}$ solutions are available to bring the body into a defined position and orientation. This high redundancy can be exploited to control the approaching angle of the (meta)tarsus limb.

Table 3. Additional Denavit-Hartenberg parameters (compare Table 2).

\begin{tabular}{ccccc}
\hline & $d_{i}$ & $\theta_{i}$ & $a_{i}$ & $\alpha_{i}$ \\
\hline $4 \rightarrow 5$ & 0 & $\theta_{5}$ & 0 & $-\frac{\pi}{2}$ \\
$5 \rightarrow 6$ & 0 & $\theta_{6}$ & 0 & $\frac{\pi}{2}$ \\
$6 \rightarrow 7$ & 0 & $\theta_{7}$ & 0 & 0 \\
\hline
\end{tabular}




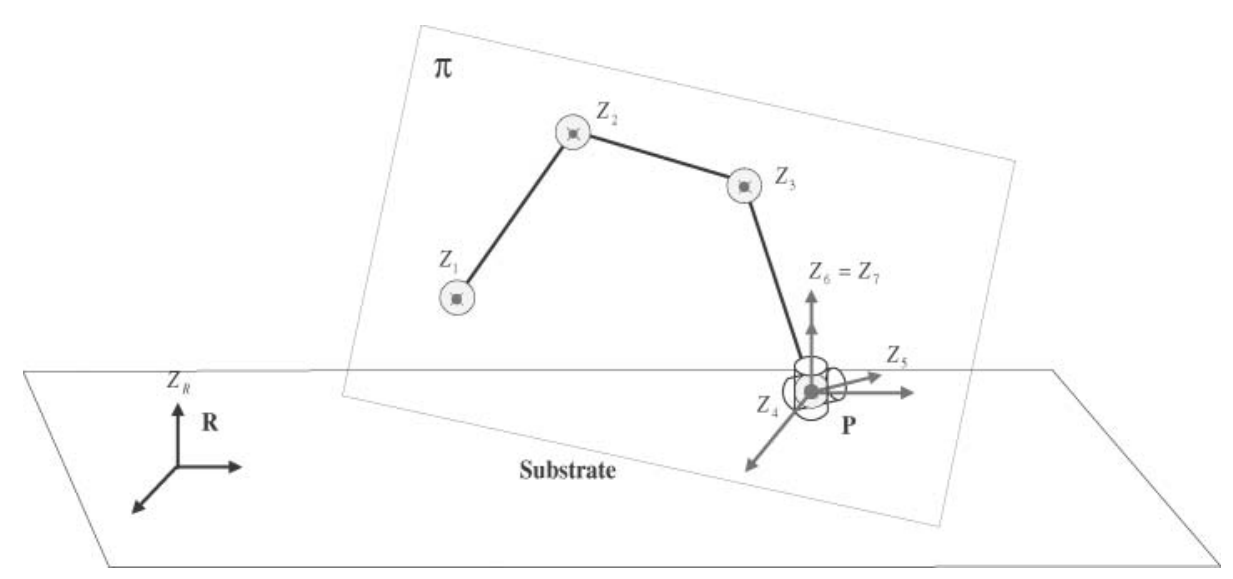

Figure 6. Model of the leg in contact.

\subsubsection{Direct kinematics}

The direct kinematics can be expressed as

$$
\mathbf{T}_{B}^{R}=\mathbf{T}_{6}^{R} \cdot \mathbf{T}_{5}^{6} \cdot \mathbf{T}_{4}^{5} \cdot\left(\mathbf{T}_{4}^{B}\right)^{-1}
$$

where $\left(\mathbf{T}_{4}^{B}\right)^{-1}$ comes from the results of the free-flight kinematics. By defining the values of the angles of the pairs, it is possible to evaluate and compute the position and orientation of the spider's body.

\subsubsection{Inverse kinematics}

The task is to search the values that must be imposed on the joints to bring the body to a desired position and orientation:

$$
\operatorname{Body}=[\mathbf{B} ; \Theta]=\left[B_{x}, B_{y}, B_{z}, \phi, \theta, \psi\right]^{\mathrm{T}},
$$

where the orientation is expressed with Euler angles. Hence, the rototranslation matrix between the reference system and the body can be expressed as

$$
\begin{aligned}
& \mathbf{T}_{B}^{R}= \\
& {\left[\begin{array}{ccc|c}
c_{\phi} \cdot c_{\theta} \cdot c_{\psi}-s_{\phi} \cdot s_{\psi} & c_{\phi} \cdot c_{\theta} \cdot s_{\psi}-s_{\phi} \cdot c_{\psi} & c \phi \cdot s_{\theta} & B_{x} \\
s_{\phi} \cdot c_{\theta} \cdot c \psi+c \phi \cdot s \psi & s_{\phi} \cdot c_{\theta} \cdot s_{\psi}-c_{\phi} \cdot c_{\psi} & s_{\psi} \cdot s_{\theta} & B_{y} \\
-s_{\theta} \cdot c_{\psi} & s_{\theta} \cdot s_{\psi} & c_{\theta} & B_{z} \\
\hline 0 & 0 & 0 & 1
\end{array}\right] .}
\end{aligned}
$$

Looking at the system structure, once the leg is chosen, the rototranslation matrix between the body and the first joint is known, and the matrix $\mathbf{T}_{0}^{R}=\mathbf{T}_{B}^{R} \cdot \mathbf{T}_{0}^{B}$ is computed. By considering that $\theta_{1}$ is the rotation that allows to move reference system 1 onto reference system 0 (Figure 6), and that the point $\mathbf{P}$ can be expressed in the coordinate system 0 as

$$
\mathbf{P}^{0}=\left(\mathbf{T}_{0}^{R}\right)^{-1} \cdot \mathbf{P}=\mathbf{T}_{R}^{0} \cdot \mathbf{P}
$$

$\theta_{1}$ can be calculated as in the free-flight condition:

$$
\theta_{1}=a \tan 2\left(p_{y}^{0}, p_{x}^{0}\right)
$$

The plane $\pi$ (Figure 6) contains the $\mathbf{Z}_{0}$ axis, the points $\mathbf{O}_{0}$ $=\mathbf{O}_{1}, \mathbf{O}_{2}, \mathbf{O}_{3}$ and $\mathbf{P}=\mathbf{O}_{4}=\mathbf{O}_{5}=\mathbf{O}_{6}=\mathbf{O}_{7}$, the contact point between the end of the (meta)tarsus link and the surface. The $\mathbf{Z}_{4}=\mathbf{Z}_{3}=\mathbf{Z}_{2}=\mathbf{Z}_{1}$ axes are orthogonal with respect to $\pi$ and can be found as follows.

The $\mathbf{Z}_{4}$ axis is the third column of the rototranslation matrix $\mathbf{T}_{1}^{R}$. The spherical joint in $\mathbf{P}$ is a virtual spherical joint that allows us to define the rotations that have to be made to put the coordinate system 7 on the coordinate system 4 . The three unknown angles are the Euler angles (ZYZ) with respect to the coordinate system 4 , and two rotations are required ( $\theta_{6}$ and $\theta_{7}$ ) to bring the $\mathbf{Z}_{7}$ axis to the known $\mathbf{Z}_{4}$ axis.

The spherical joint rototranslation matrix is

$$
\mathbf{T}_{7}^{4}=\mathbf{T}_{5}^{4} \cdot \mathbf{T}_{6}^{5} \cdot \mathbf{T}_{7}^{6}
$$

The $\mathbf{Z}_{4}^{7}$ axis can be expressed as

$$
\mathbf{Z}_{4}^{7}=\mathbf{T}_{R}^{7} \cdot \mathbf{Z}_{4}^{R}
$$

equal to the third column of the transposed matrix $\left(\mathbf{T}_{7}^{4}\right)^{\prime}$. Hence, the $\mathbf{Z}_{4}^{7}$ is

$$
\mathbf{Z}_{4}^{7}=\left[-s_{\theta_{6}} \cdot c_{\theta_{7}}, s_{\theta_{6}} \cdot s_{\theta_{7}}, c_{\theta_{6}}, 1\right]^{\mathrm{T}}
$$

and, by comparing the two expressions for $\mathbf{Z}_{4}^{7}$, the angles $\theta_{6}$ and $\theta_{7}$ can be found. The remaining planar four-bar linkage includes the pairs that define $\theta_{2}, \theta_{3}, \theta_{4}$ and $\theta_{5}$.

This articulated mechanism has 1 DOF and admits $\infty^{1}$ solutions. The configuration is chosen to assure conditions on the overall adhesion of the leg and the system. Hence, as the points $\mathbf{O}_{1}$ and $\mathbf{O}_{5}$ are known, this DOF is used to 


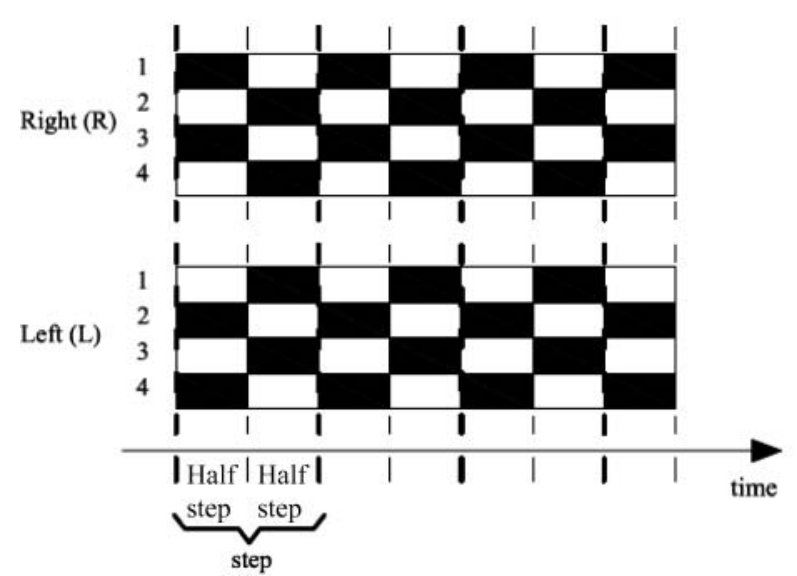

Figure 7. Ideal stepping pattern: Alternating tetrapod.

impose the approaching angle through $\theta_{5}$. Once this angle is chosen, the spherical wrist is defined and the $\mathbf{O}_{3}$ origin becomes available.

The remaining problem is an inverse kinematics of an $\mathrm{R}-\mathrm{R}-\mathrm{R}$ dyad, the same already computed for the free-flight configuration.

\section{Gait patterns}

Legged locomotion requires highly coordinated movements of the individual legs, especially when the legs' individual working ranges overlap. This is even more the case in an animal (and robot) using eight legs simultaneously. In order to achieve directed locomotion of the overall system, each leg also has to perform a different movement. Generally, the first and fourth leg pair (seen from the front) move mostly in the vertical plane (extension - retraction), while the second and third leg pair move rather in vertical and horizontal directions combining a torsional movement with a flexing (second pair of legs) or extending (third pair of legs) action. The first and second pair of legs are directed forward, i.e. lifted and extended, and then they pull with a flexion motion providing the propulsive force. The third and fourth leg pairs are pointed backward and push.

The studies on spider locomotion (Wilson 1967; Shultz 1987; Foelix 1996; Weihmann 2008) were carried out on a flat surface in the horizontal plane. Since data on vertical locomotion in spiders are missing, we assumed similar behaviour as reported from cockroaches (Goldman et al. 2006). Furthermore, it can be assumed that on vertical planes the first and second leg pairs generate negative normal force producing counter torque, while the necessary shear force is mostly produced by the last two leg pairs. In a first approximation, the basic stepping pattern of spiders is similar to the patterns of other arthropods, i.e. a functional biped where four legs (e.g. L1, R2, L3, R4 and R1, L2, R3,

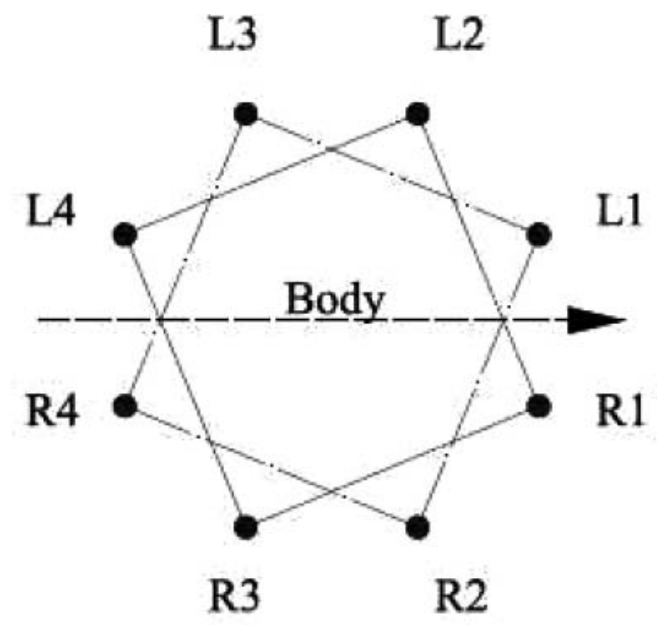

L4) move in phase, while the other four act with a phase shift of approximately 0.5 (Foelix 1996). A basic sketch of this gait - called alternating tetrapod - is shown in Figure 7. The tetrapod configuration allows for high static stability as on level ground the center of mass of the system is inside the tetrapod area.

In reality, the spiders' legs not are perfectly synchronised. Indeed, the feet L1 - R2 - L3 - R4 (like in the other half-step the R1 - L2 - R3 - L4) do not touch the surface exactly at the same time but in a metachronal way from caudal to cranial that means that the sequence becomes like a wave initiating with the hind legs (Wilson 1967). With increasing velocity this asynchronicity diminishes to zero. In addition, we find double support phases, i.e. when both tetrapods are in ground contact at the same time. These features, however, facilitate locomotion control as they bridge mechanically unstable instants at the interface between free flight and ground contact. In addition, double support phases may play an important role in the control of adhesion during vertical or overhead locomotion.

\section{Implementation in a simulator}

We implemented a kinematic simulator in Matlab ${ }^{\mathrm{TM}}$ using the above-described kinematic model of the spider as well as the alternating tetrapod locomotion pattern and the approaching adhesion condition (Figure 8).

If a no gravity environment is considered, in all the slope conditions the adhesion of all eight legs is important in order to maintain the robot in contact with the substrate, hence the adhesive kinematic condition has to be maximised. On the other hand, if the considered environment features gravity, the adhesion requirements can conceptually change with the slope. This means that, if a flat plain surface is considered, the adhesive condition can be relaxed and the trajectories followed by the legs do not 

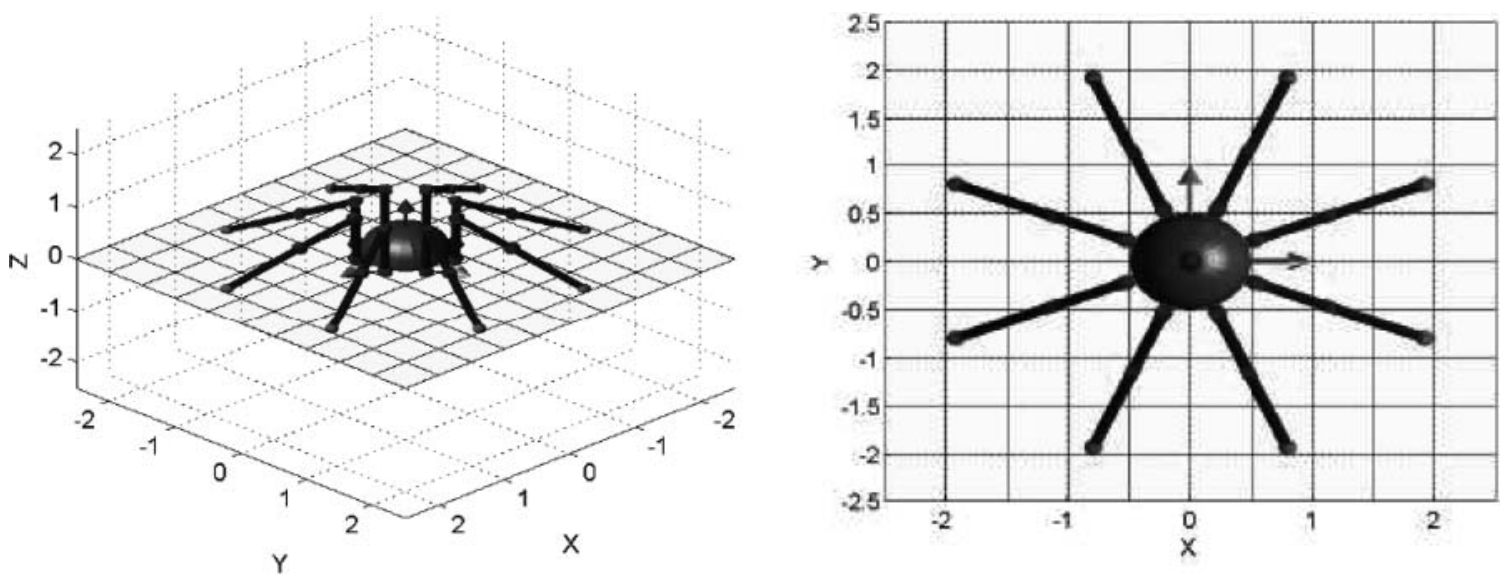

Figure 8. Spider-robot simulator.

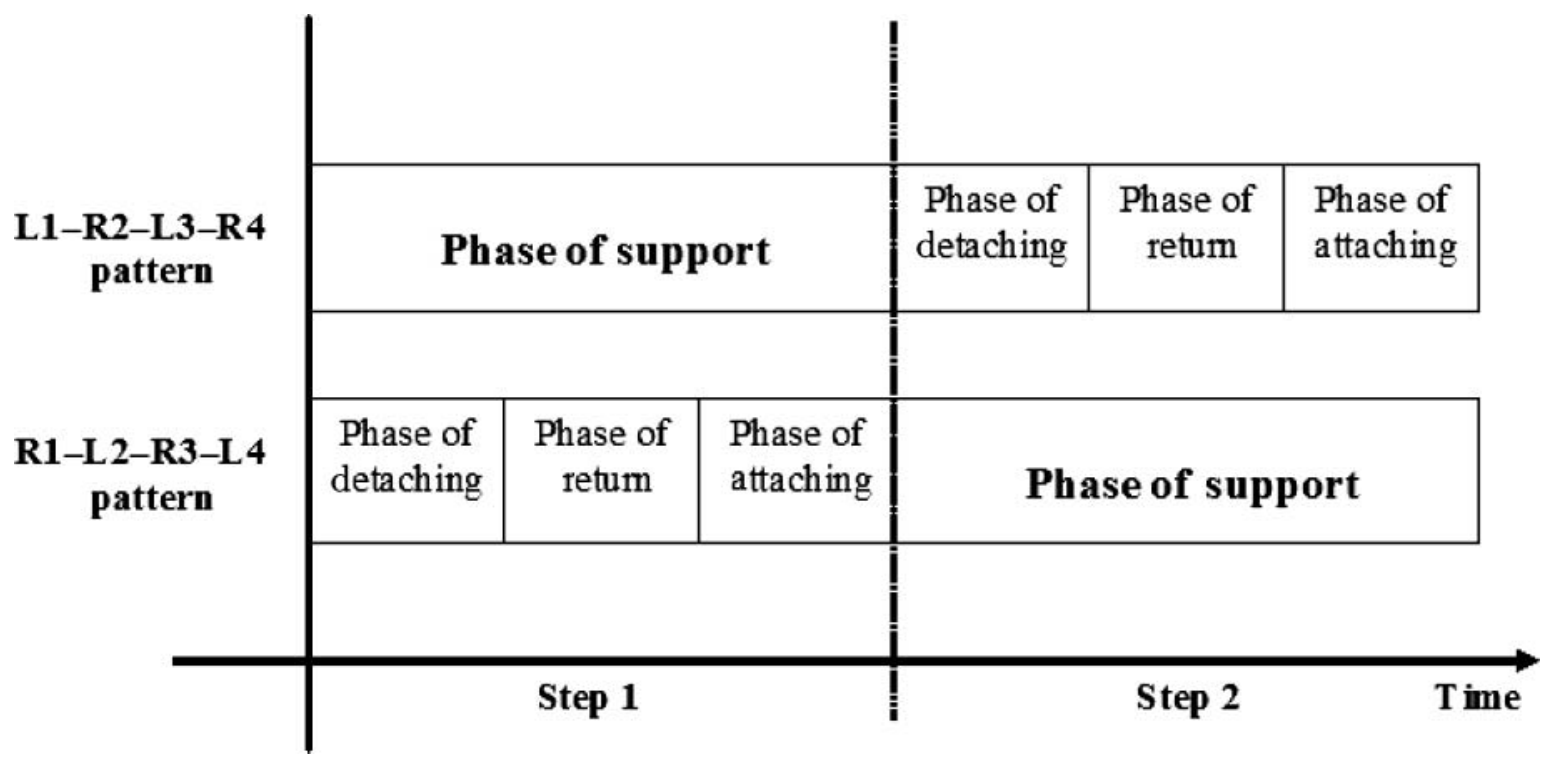

Figure 9. Fundamental step (1: first-half; 2: second-half).

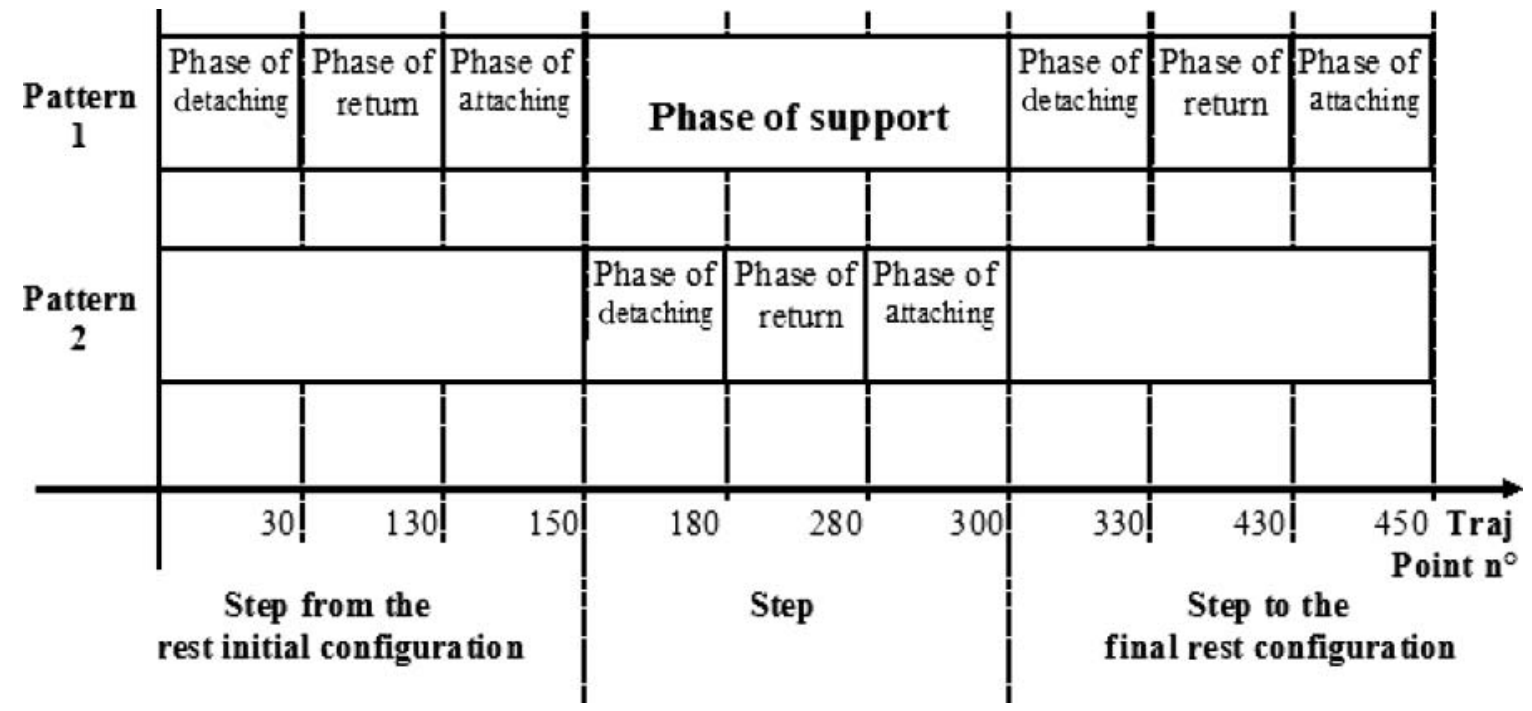

Figure 10. Phases subdivision with respect to the number of trajectory points. 


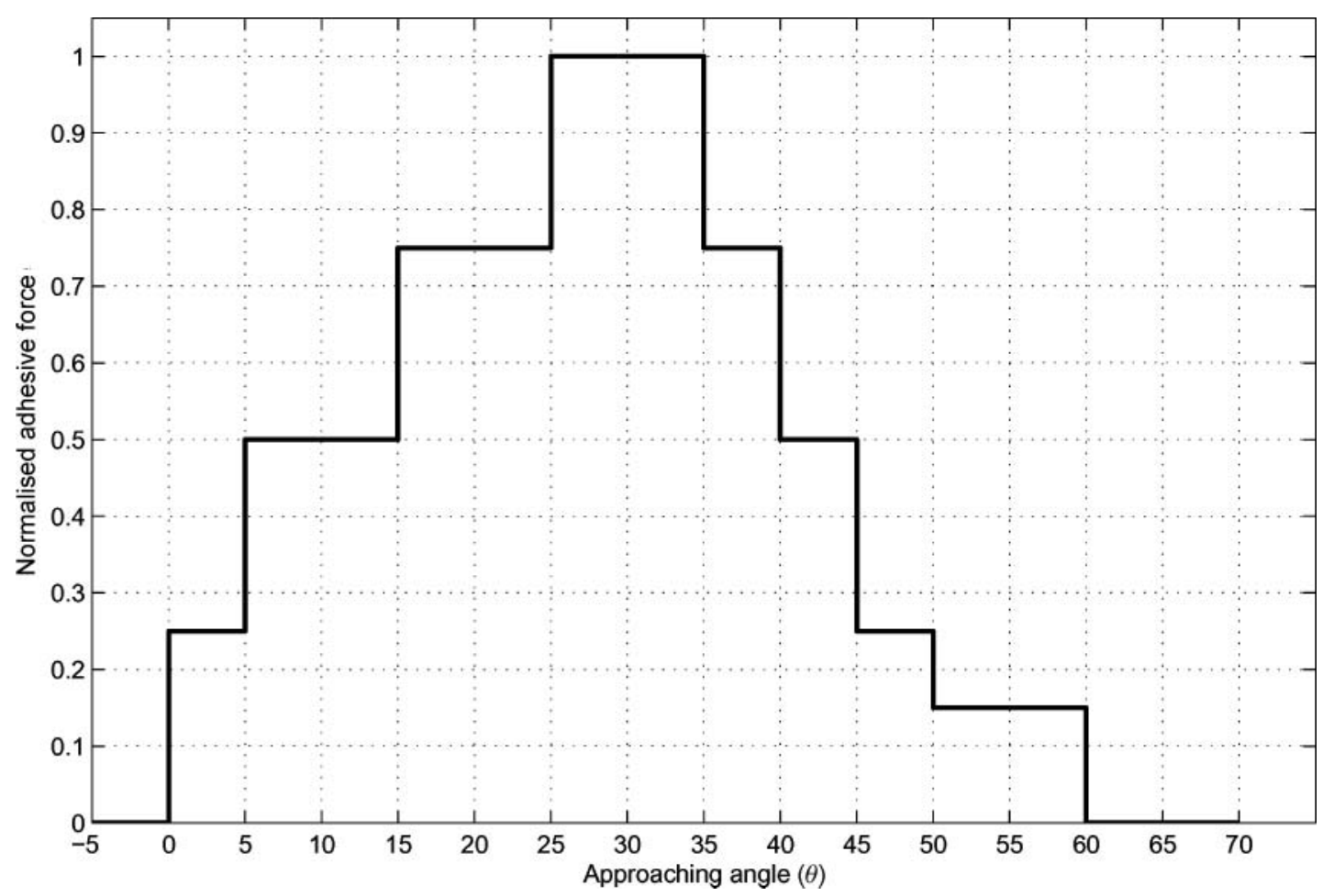

Figure 11. Discretised and normalised adhesive force.

have to satisfy or to warrant a safety factor. Besides, if the walk on an inverted flat surface is simulated, a suitable safety factor has to be maintained for all the locomotion. By considering a no gravity environment, for all the simulated locomotion the trajectories followed by the legs and the body must allow a suitable degree of safety.

The trajectory, referred to the body, is split in fundamental steps. The fundamental step is the elementary distance

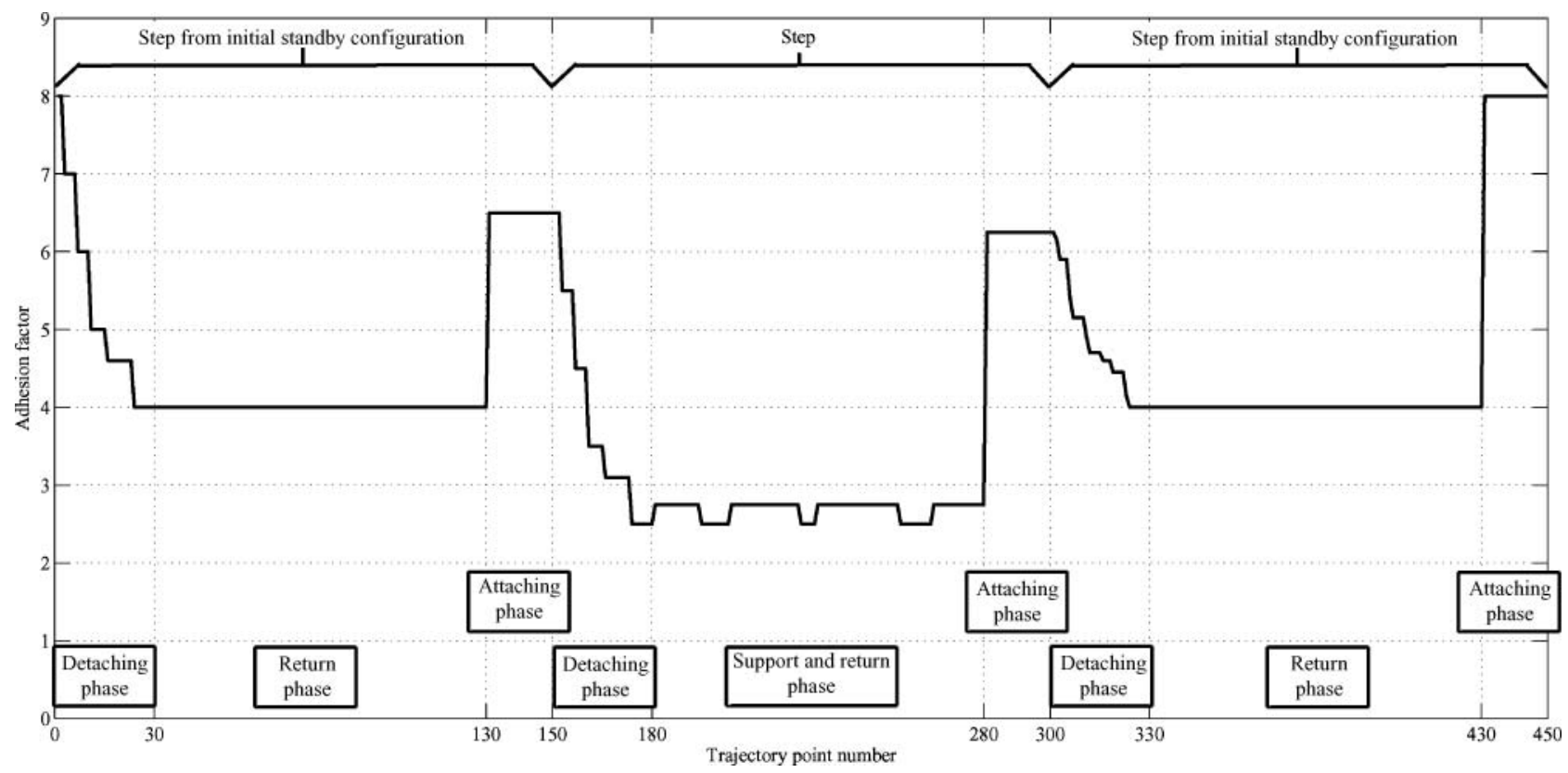

Figure 12. Adhesion factor with respect to the trajectory points. 


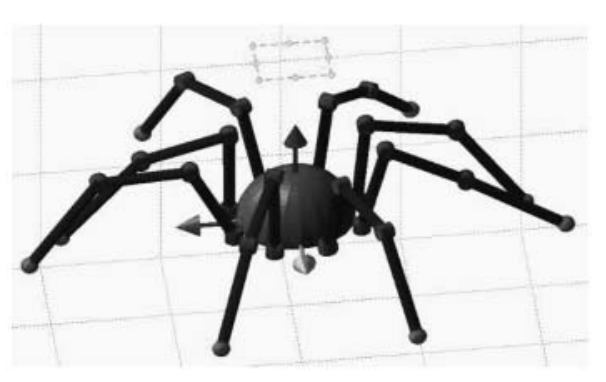

(a)

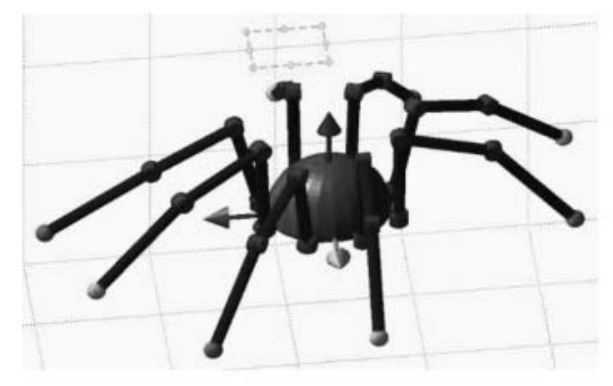

(c)

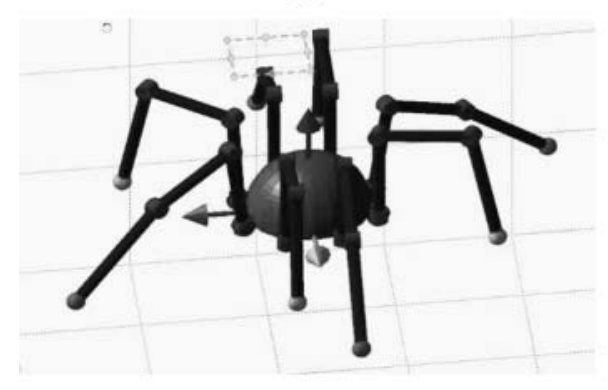

(e)

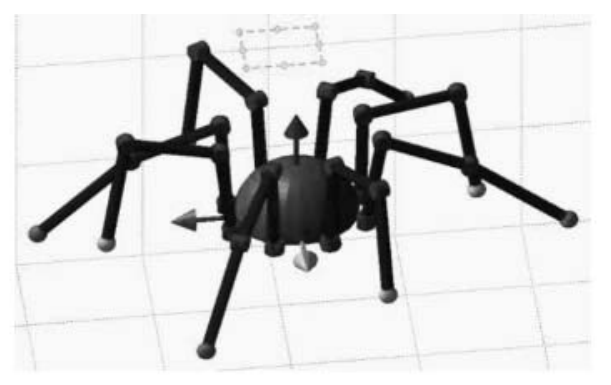

(b)

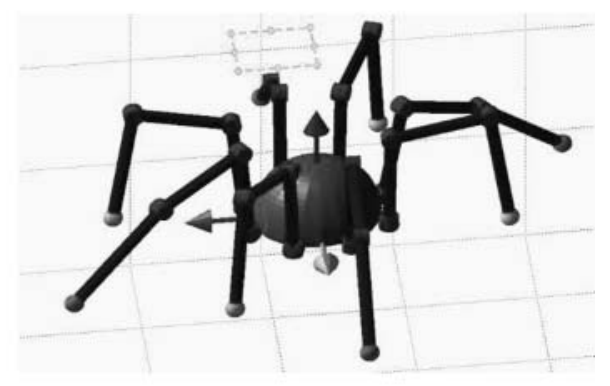

(d)

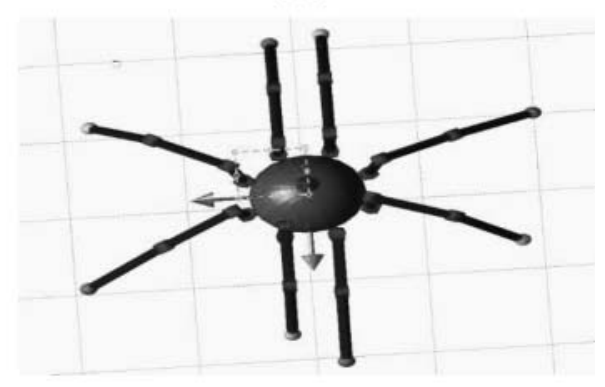

(f)

Figure 13. Spider-robot simulation: (a) global view (in line-dot the target square run), setting of the step, eight legs in contact; (b) first-half of the step, four legs in contact and four in flight; (c) end of the first-half step. Eight legs in contact, four in attaching; (d) second-half step; (e) rotation of the four legs in flight; (f) end of the step. The tip of the (meta)tarsus of each leg is visualised with different colours due to the angle of adhesion and to the condition of the leg (i.e., adhesion contact, flight).

the system can run in a single step and that can be suitably chosen with respect to the imposed task. Every step and footfall position are computed for maintaining the height of the body constant and ensuring an optimal range of possible substrate-approaching angles for the tarsus.

At every half-step, the kinematic simulator switches between two states: the supporting and the motion conditions. In the support phase, the legs are in the best adhesion condition (approaching angle between $25^{\circ}$ and $35^{\circ}$ ) and support the body during the fundamental translation. The motion phase is divided into three elements (Figure 9), the return phase and the two changes of attachment. The first element is the detaching phase. The legs are in contact and an approaching angle (i.e., the angle between tarsus and substrate) is in the range of $20^{\circ}-40^{\circ}$. For detaching the legs correctly, the above-mentioned angle is increased up to the detaching threshold, at least greater than $70^{\circ}$, and the adhesive force is minimised. Subsequently follows the return phase. The four legs of one functional tetrapod are lifted up from the substrate, retracted near the body, rotated about the first revolute pair of the kinematic chain and finally extended to reach the correct position for the subsequent support phase. Then, the next position of each leg is calculated and implemented taking into account the behaviour that the leg has to follow and the direction of locomotion. Moreover, the next positions are studied to allow the inverse kinematic solutions and avoid collisions between the legs. Finally follows the attaching phase where the legs are 
already in contact with the surface but the approaching angle can be out of the optimal range of adhesion. In this case, the system has to maintain the contact and work on the angle between the (meta)tarsus and the surface to reach an optimal adhesion. Following this, a small proximal movement of 5-10 $\mu \mathrm{m}$ to adequately activate the attaching elements is implemented.

In order to choose a correct configuration for each leg, the overall adhesion of the system has to be taken into account. Indeed, in the implemented model the overall estimated adhesion of the model, based on a discretisation of the adhesion curve described in (Gao et al. 2005) and a suitable safety factor, can be chosen and controlled at each instant.

The following example shall demonstrate the effectiveness of the system by simulating the adhesion factor with respect to the points of the leg's trajectory. The spider-robot starts from the resting condition (i.e., standby configuration), performs a fundamental step and, finally, returns to the resting position. The number of trajectory points into which each phase is subdivided are as follows:

- supporting phase: 100

- return phase: 100 ;

- detaching phase: 30 and

- attaching phase: 20.

In Figure 10, the phase subdivisions that compose the example are defined with respect to the trajectory points.

Figure 11 shows the discretisation of the normalised adhesive force with respect to the approaching angle based on Gao et al. (2005) results. The sum of the discretised adhesive factors of the legs (for each leg, a discretised adhesive factor between 0 and 1 directly related to the approaching angle has been assumed) in contact with the substrate for each point of the trajectory is displayed in Figure 12.

It can be easily underlined that, with this solution, the most critical situation occurs when four legs are in contact (i.e., support phase) and four are in flight (i.e., return phase). Moreover, during a support phase the body is shifted, hence the approaching angle of the legs in contact can change as the overall adhesion. The overall adhesion coefficient results $\geq 2.5$ during all the step. As previously said, in an ideal condition a spider E. arcuata can show a safety factor bigger than 160 (Kesel et al. 2004) and, hence, a mean value of 20 for each leg. Thus, in ideal conditions, for each single point of the implemented trajectory, if the dimensions of the $E$. arcuata are considered, a minimum safety factor of 50 is guaranteed.

By looking at the adhesion factor in the rest condition, it is possible to underline how this position has been accurately chosen in order to allow the maximum contribution to the adhesion from each leg.

As a visual output, an example of the locomotion simulation is reported in Figure 13. the initial configuration is the symmetrical position at rest. The simulations show the effectiveness of the model. During each step, the overall adhesion and also the desired point of contact can be suitably controlled by studying the adhesive factor of each leg. Moreover, the configuration and disposition of the legs allow us to improve the capabilities of the model (thanks to the symmetrical condition that can be exploited for evaluating and choosing the best path).

\section{Summary and outlook}

Freely hunting spiders such as E. arcuata combine high locomotory mobility with subsequent adhesion and detachment at a low energetic cost. The asymmetry between tight attachment and easy detachment results from an asymmetry of the setulae tips and dedicated control of the angle between tarsus and substrate. The present study demonstrates that the same result in control can be achieved in a system with drastically reduced kinematic complexity compared to the animal model. The kinematic simulator shows reliable locomotion on both even and inclined surfaces, with constant control of a (theoretical) passive attachment device according to the model of E. arcuata.

In order to achieve a first working version of the kinematic simulator, its tasks were restricted to locomotion in combination with low energetic control of attachment. To this end, it was necessary to extrapolate the attachment characteristics from the gecko's setulae rather than the spider's, since the former have been studied in greater detail. The angles between tarsus and substrate during attachment and detachment also await experimental validation. By considering that the adhesion increases not only when a normal load, but also when a parallel load is applied (Niederegger and Gorb 2006; Autumn et al. 2000), we argue that in spiders not only a normal pre-load but also a parallel-sliding movement is employed in order to enforce contact between the attaching elements and the surface, as demonstrated for geckos (Autumn et al. 2000). Other peculiarities of the spider locomotory system are not integrated at the current state. For example, different lengths of adjacent legs allow for bigger angular movements without collision of the legs. This would enhance the maximum velocity of the technical spider. Weihmann (2008) also reports on three gaits for different velocities, which may be of interest for a robotic implementation also.

Finally, the simulator awaits extension to perform complex tasks such as swapping between two differently inclined surfaces, e.g. changing from an even substrate to a vertical one or bridging gaps in the substrate. In order to be a functional robot, but also to profit from the capabilities of the spider locomotory apparatus, our model needs to become able to grab and handle objects with its first leg pair by adapting the kinematics in order to exploit the attachment devices at the tarsi. Finally, we will evaluate the 
potential to integrate dynamic behaviours such as jumping and landing into the existing architecture.

At the present state, the kinematic simulator is able to control the locomotion with reference to the spider's overhead position, on inclined substrate and in micro-gravity by successfully controlling the attachment to the substrate via leg kinematics. Hence, the simulator does not require a dedicated control system for the attachment, therefore displaying comparatively low complexity.

The conceived layout including leg geometry offers some principal advantages. In future stages, it is potentially capable of locomotion and attachment on non-coplanar surfaces and also might become able to handle large objects using the first leg pair.

In a forward strategy, future work will deal with the evaluation of several aspects, such as rotational and axial symmetry of the concept, morphology-depending leg adaptations, dynamic analysis and locomotion, coping with noncoplanar surfaces, grasping and manipulating free-floating objects.

\section{Acknowledgement}

This project was supported by the ARIADNA scheme (study ID 06/6201) of the European Space Agency.

\section{References}

Autumn K, Liang YA, Hsieh ST, Zesch W, Chan WP, Kenny TW, Fearing R, Full RJ. 2000. Adhesive force of a single gecko foot-hair. Nature 405:681-685.

Bellingham JG, Rajan K. 2007. Robotics in remote and hostile environments. Science 318(5853):1098-1102.

Betz O, Kölsch G. 2004. The role of adhesion in prey capture and predator defence in arthropods. Arthropod Struct. Dev. 33(1):3-30.

Ellis CH. 1943. The mechanism of extension in the legs of spiders [master's thesis]. Gradute School of California Institute of Technology. Pasadena, USA.

Foelix RF. 1996. Biology of spiders. 2nd ed. Oxford University Press: Oxford, UK.

Gao H, Wang X, Yao H, Gorb S, Arzt E. 2005. Mechanics of hierarchical adhesion structures of geckos. Mech. Mater. 37(23):275-285.

Goldman DI, Chen TS, Dudek DM, Full RJ. 2006. Dynamics of rapid vertical climbing in cockroaches reveals a template. J. Exp. Biol. 209:2990-3000.

Hirose S, Kawabe K. 1998. Ceiling walk of quadruped wall climbing robot NINJA-II. Proceedings of the 1st International Conference on Walking and Climbing Robots (CLAWAR 98); Brussels, Belgium.

Huang J-N. 2004. Taxonomic study of Myrmarachne (Araneae: Salticidae) from Taiwan [master's thesis]. National Sun Yat-sen University, Taiwan.

Karner M. 1999. Volumenverschiebungen beim Sprung der Jagdspinne Cupiennius salei [Ph.D. thesis]. Johann Wolfgang Goethe-Universitt Frankfurt am Main, Biowissenschaften.
Kesel AB, Martin A, Seidl T. 2003. Adhesion measurements on the attachment devices of the jumping spider Evarcha arcuata. J Exp Biol. 206:2733-2738.

Kesel AB, Martin A, Seidl T. 2004. Getting a grip on spider attachment: an AFM approach to microstructure adhesion in arthropods. Smart Mater Struct. 13:512-518.

Kim S, Spenko M, Trujillo S, Heyneman B, Mattoli V, Cutkosky MR. 2007. Whole body adhesion: hierarchical, directional and distributed control of adhesive forces for a climbing robot. Proceedings of the IEEE International Conference on Robotics and Automation (ICRA 2007); Apr 10-14; Roma, Italy.

La Rosa G, Messina M, Muscato G, Sinatra R. 2002. A lowcost lightweight climbing robot for the inspection of vertical surfaces. Mechatronics 12(1):71-96.

Menon C, Murphy M, Sitti M. 2004. Gecko inspired surface climbing robots. Proceedings of the IEEE International Conference on Robotics and Biomimetics (ROBIO 2004); 22-26 August; Kunming, China, pp. 431-436.

Menon C, Sitti M. 2005. Biologically inspired adhesion based surface climbing robots. Proceedings of the IEEE International Conference on Robotics and Automation (ICRA 2005); 18-22 April; Barcelona, Spain.

Nachtigall W. 1974. Biological mechanisms of attachment. Berlin: Springer.

Niederegger S, Gorb S. 2006. Friction and adhesion in the tarsal and metatarsal scopulae of spiders. J. Comp. Physio. 192:12231232.

Parry DA. 1957. Spider leg-muscles and the autotomy mechanism. Q J Microsc Sci. 98:331-340.

Sciavicco L, Siciliano B. 2001. Modelling and control of robot manipulators. London, GB: Springer.

Sensenig AT, Shultz JW. 2002. Mechanics of cuticular elastic energy storage in leg joints lacking extensor muscles in arachnids. J Exp Bio. 206:771-783.

Sensenig AT, Shultz JW. 2004. Elastic energy storage in the pedipalpal joints of scorpions and sun-spiders (Arachnida, Scorpiones, Solifugae). J Arachnol. 32:1-10.

Shultz JW. 1987. Walking and surface film locomotion in terrestrial and semi-aquatic spiders, J Exp Biol 128:427444.

Unver O, Uneri A, Aydemir A, Sitti M. 2006. Geckobot: a gecko inspired climbing robot using elastomer adhesives. Proceedings of Robotics and Automation (ICRA 2006); New Orleans, LA, USA, pp. 2329-2335.

Weihmann T. 2008. Biomechanische analyse der ebenen lokomotion von Ancylometes bogotensis (Keiserling, 1877) (Chelicerata, Arachnida, Lycosoidea) [Ph.D. thesis]. FriedrichSchiller-University, Jena.

Wilson DM. 1967. Stepping patterns in tarantula spiders. J Exp Biol. 47:133-151.

Yano T, Suwa T, Murakami M, Yamamoto T. 1997. Development of a semi self-contained wall climbing robot with scanning type suction cups. Proceedings of the IEEE International Conference on Intelligent Robots and Systems (IROS'97); Sep 7-11; Grenoble, France.

Yoshida H, Tso I, Severinghaus LL. 2000. The spider family Theridiidae (Arachnida: Araneae) from Orchid Island, Taiwan: descriptions of six new and one newly recorded species. Zool Stud. 39(2):123-132.

Xu Z, Ma P. 2002. A wall-climbing robot for labeling scale of oil tank's volume. Robotica 20(2):209-212. 

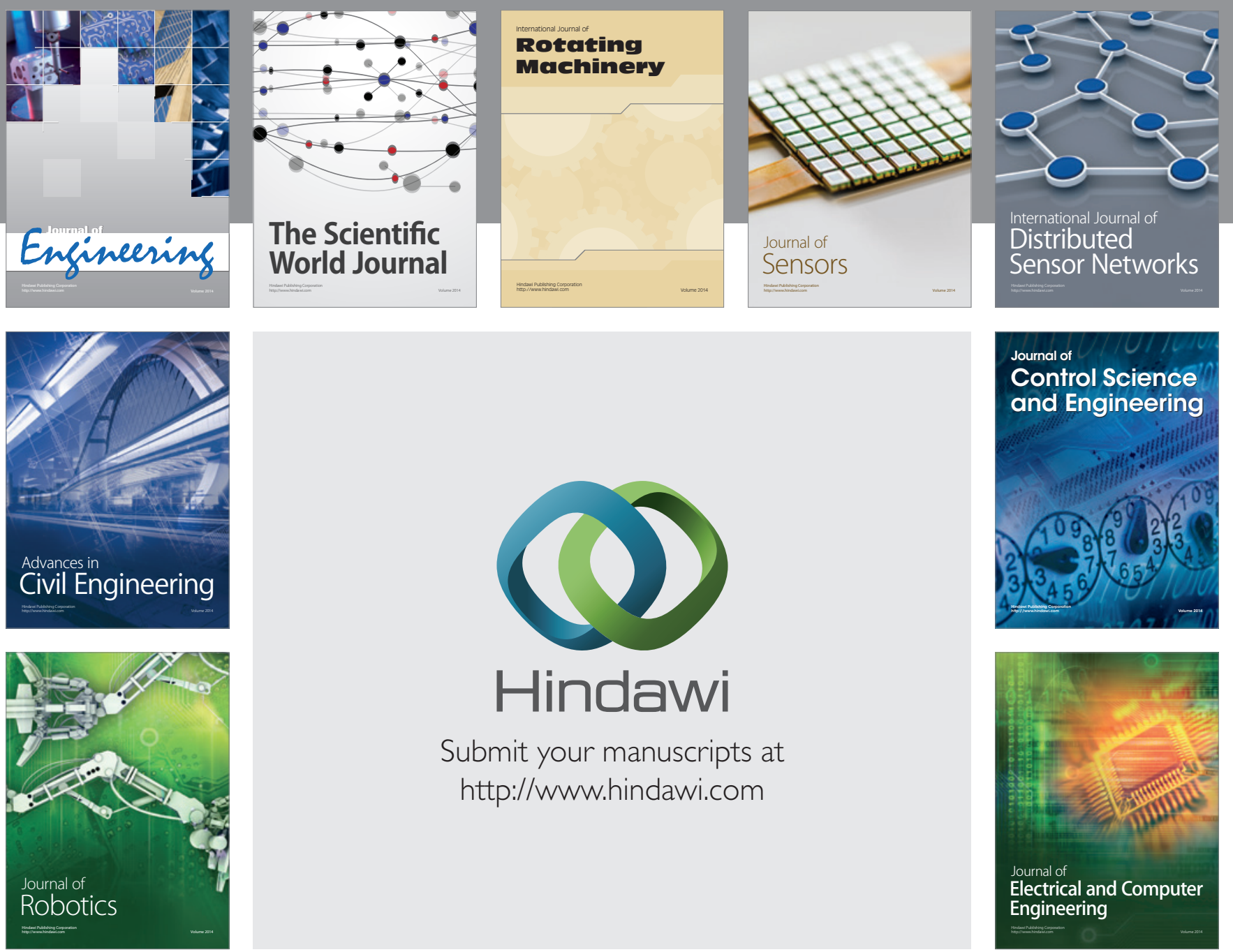

Submit your manuscripts at

http://www.hindawi.com
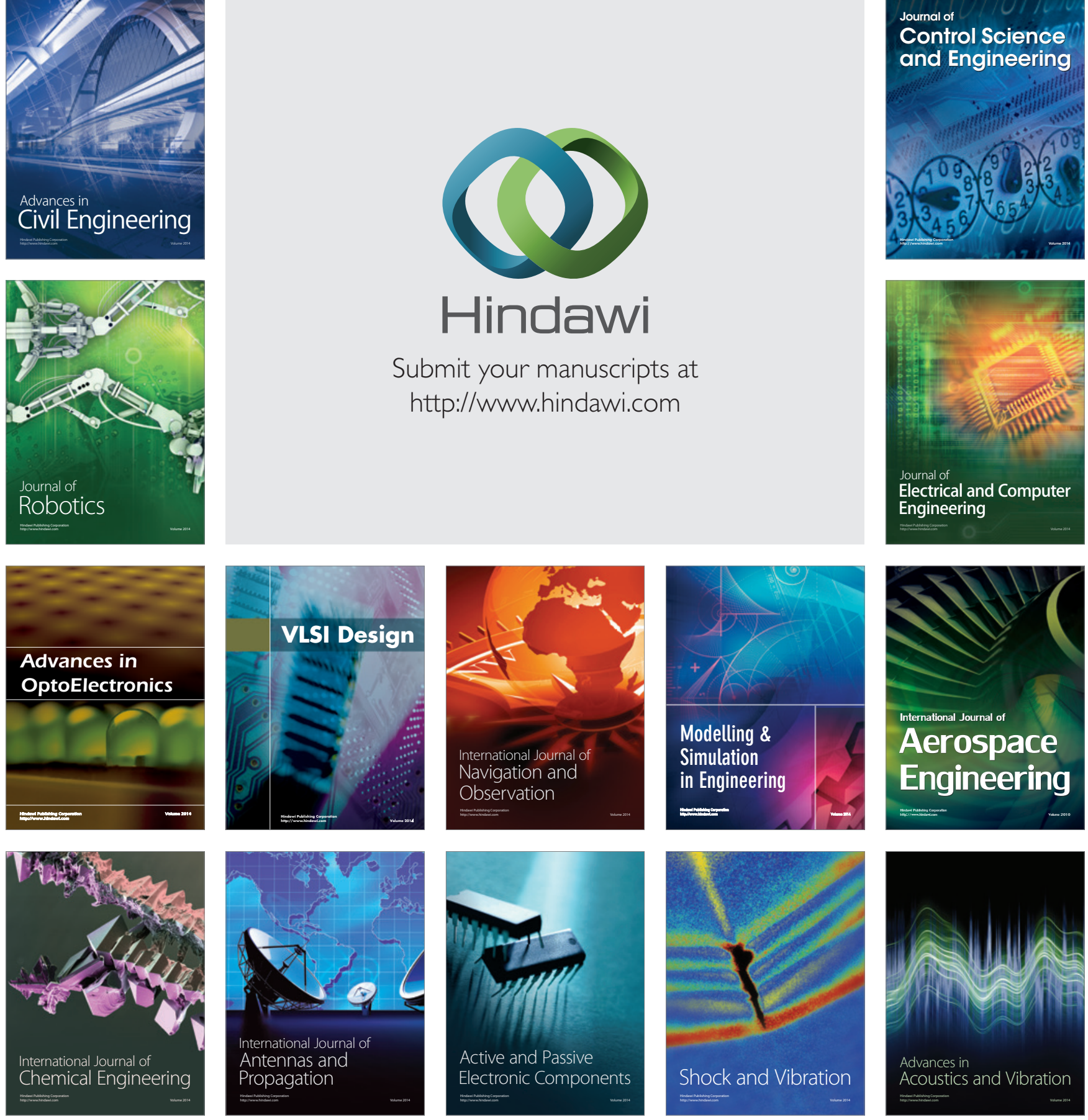